\title{
The New Development of CAD in Clothing
}

\author{
Lingxia Zhang, Hongzhi Zhang \& Yinglin Li \\ Tianjin Polytechnic University \\ Tianjin 300160, China \\ E-mail:zlingxia2006@gmail.com
}

\begin{abstract}
This text gives introduction to the history and the status quo of CAD/CAM in clothing and puts forward some questions. It also proposes a neural network model of artificial intelligence, which indicates the direction of intellectualized and three-dimensional development of clothing by use of neural network.

Keywords: Digital clothing, Artificial intelligence, Visible technology

In 1959, CAD (computer aided design) as a conception was proposed. But since it was introduced into the clothing industry in 1970s, it has accelerated to change the traditional manual way of production. At the same time, CAD has been developing at a surprising speed. At present, the application of the computer has involved in various clothing fields of design, manufacture, sales, management, and education. It has included the whole process of clothing production.

\section{The development of digital clothing technology.}

In 1970s, the mature CAD in clothing proposed on personal computers has laid a foundation for a wide range of application.

In 1980s, CAM (computer Aided Manufacturing) and FMS (flexible manufacturing system) further improved the key technologies relevant to $\mathrm{CAD}$ in clothing.

In 1990s, the development of computer information management technology promoted the integration of design, manufacture and management. And clothing CIMS (computer integrated manufacturing system) has come into being.

Nowadays, the new technologies are undergoing a revolutionary change. Made-to-measure, three-dimensional computer simulation and visualization have become the important study programs of the clothing digital technology.
\end{abstract}

Now a variety of CAD systems have been widely used in the field of clothing. Lectra system gives a comparative concentration on the integration of CAD software. Since 1998, it has adopted new strategies to expand its technology and provided a system of simulation for the entire textile industry from yarn and textile design to the manufacture of virtual goods and to the ready-to-wear. It represents the direction of development of a new generation of textile and clothing CAD.

Assyst system can simulate three-dimensional effect and give the design of structure and fabric. It has more than 400 kinds of databases for optional format-making, picturizing and modifying. It can make format automatically or according to the actual size and change the size freely. The system also has intelligent database, which can make management of styles quick and efficient.

Spanish Investronica system has made effective efforts in the application of artificial intelligence and other technologies. Its CAD / CAM system is featured with a sound cost management system, production-o-rial system, and warehouse management system. After a customer selects the style and the material, it measures the customer, then makes an automatic sampling design, changes the size, arranges the material, makes autonomous single cutting out, and finishes the final step of the ready-to-wear. It is really a highly automated customer-oriented system.

\section{The limitations of clothing CAD technology.}

In recent years, there have existed some problems in the rapid development of the CAD technology. For example, the development of cell technology is overstressed; too much emphasis is on the general system while the design of product life cycle in the process, the development of net application and database, and feature bank are neglected.

Second, CAD is lack of software features and effects of humanity, intelligence, and visualization. Although some software can be used for the three-dimensional garment paper pattern design at present and can simulate a stronger real wearing effect, if the designer feels dissatisfied, it must return to the plain plate for further modification. Such repetition is bound to lose the advantages of CAD technology in the users' eyes. 


\section{The new development of apparel CAD Technology.}

To make apparel CAD system more humane, more convenient and more efficient to be accepted by the designers, we tries to propose a more humane process-oriented intelligentized artificial neural network garment CAD technology. Its principle is to, on the basis of the 3-dimensional body measurement, design the clothing pieces according to the data measured, and turn the two-dimensional pieces into three-dimensional ready-to-wear, and making it simulate the motion of human beings, as shown in Figure 1.

Then through artificial neural networks, each human act will be put into the computer. The size degree of each body part will be decided and then a change will be made of the ready-to-wear model. Finally the model will be seen as two-dimensional clothing pieces.

Artificial neural network function can adopt the simple RBFNN(radial basis function neural network), which has such advantages as the strong ability of nonlinear approximation, the simple network structure, the linear net weights and output, fast learning speed, and good visual effect. It has been widely used in pattern recognition, function approximation, signal processing, system modeling, intelligent control, and other fields. RBFNN is shown in Figure 2, including an input layer, a hidden layer and an output layer. There is only one output node, indicating the degrees of fitness in GA. Its value is from 0 to 1 .

For a given training set, RBF neural network can approximate the function relationship in it. By using gradient descent training, RBF neural network parameters can be obtained, including RBFNN's centre, the width and the output weight. At the initial stage when the system begins to run, the movement of every part is grouped into the random design programs. Each program means the corresponding state of the parts when body moves and clothing fitness degrees. Professional designers will judge the various programs and give the evaluation. Such evaluation value is the fitness degrees of the apparel. A set made up of groups of such relations can be used for training RBF neural network. The parameters of RBF network is the fitness degree function of different parts. With this function every part of the clothing fitness degree can be calculated.

\section{Conclusions.}

This text has introduced the development process of CAD technology and its future direction of growth. It has also proposed an intelligent artificial neural network garment CAD system, which emphasizes the humanity, automation, and integration of the design. Its intelligence supports self-teaching, self-organization, self-adapting, self-correcting, and parallel searching, associative memory, pattern recognition, automatic acquisition of knowledge, and other technologies. The methods used have been also studied in the medical and many other fields.

At present, the system still exist such problems as numerous calculation, poor simulation effect of the apparel with complex structure. In the clothing area, the development and application of three-dimensional CAD still lag behind other fields. To achieve the conversion from two-dimensional to three-dimensional clothing CAD, a lot of problems should be solved such as the fabric texture and dynamic performance, three-dimensional reconstruction, vivid and flexible surface modeling, and the conversion from three-dimensional garment design model to two-dimensional plain clothing pieces, and many other technical issues, which has resulted in the long cycle of developing three-dimensional garment $\mathrm{CAD}$ and more technical difficulty. But it represents the future direction of development of apparel CAD and has a good prospect for further growth.

\section{References}

Gray, Stephen. (1998).CAD / CAM in clothing and textile. Gower publishing Limited.

Hagan, Martin T. Demuth, Howard B. and Beale, Mark H. (2002). Trans. by Dai Kui. Neural Network Design. China Machine Press.

Jiang, Zongli. (2001). Introduction to Artificial Neural Network. Higher Education Press.

Qiu, Peiqiong and Chen, Jihong. (2005). Talking about the development of domestic garment CAD/CAM system. Shandong Textile Science and Technology. Vol.1, pp. 40-42.

Wen, Lisheng. (1998). The use and growth of garment CAD/CAM in China. Textile Herald. Vol. 1, pp.45-47.

Yang, Qin-pu., Sun, Bingnan. And Jin, Hu. (2006). The application of artificial neural network in civil engineering. Hypothermia Construction Techniques. Vol. 6 (114), pp. 64-65.

Zhao, Nuoping, Zhang, Weiyuan. and Zhang, Hongzhi. (2000). The development and prospect of apparel CAD technology. Tianjin Textile Engineering Journal. vol.19 (5), pp.70-73. 


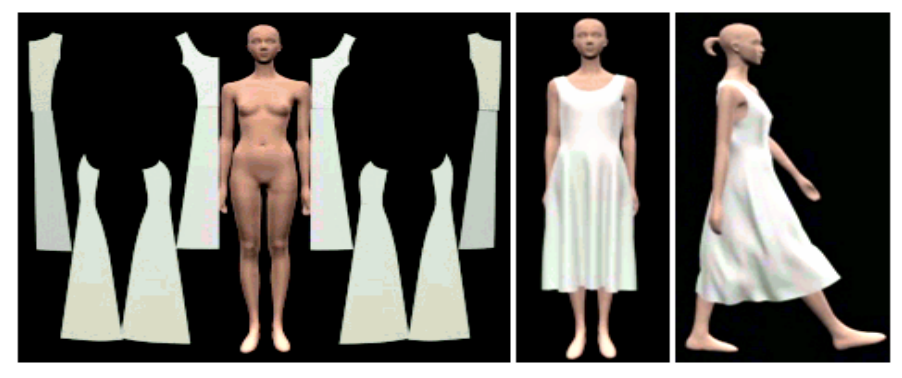

Figure 1. The virtual design effect of apparel

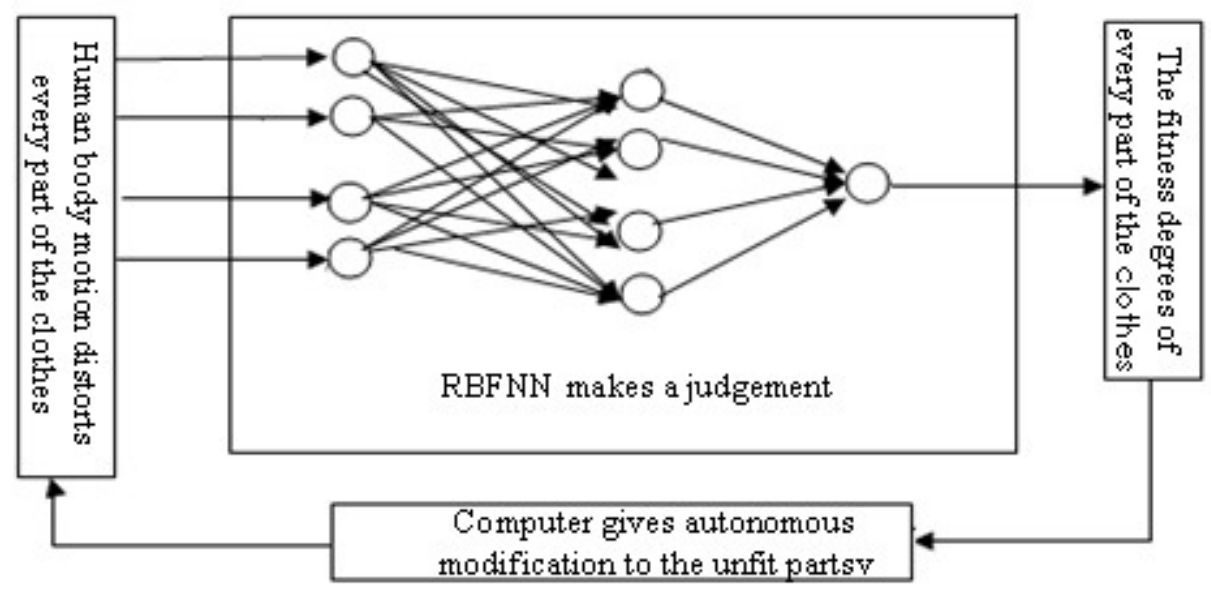

Figure 2. The application theory of neural network system 